\title{
KIAA1199 as a potential diagnostic biomarker of rheumatoid arthritis related to angiogenesis
}

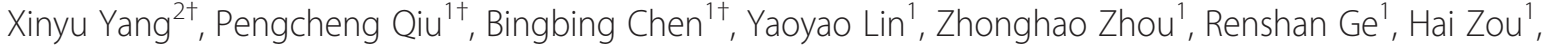 \\ Jianmin Wang ${ }^{3^{*}}$ and Jianguang Wang ${ }^{1 *}$
}

\begin{abstract}
Introduction: Our previous proteomic study on fibroblast-like synoviocytes (FLSs) derived from the synovial tissues found that the expression of KIAA1199 was higher in rheumatoid arthritis (RA) patients than in healthy controls. The aim of this study was to examine the biological function of KIAA1199 and evaluate its clinical diagnosis value in RA.

Methods: The over-expression of KIAA1199 was verified by quantitative real-time polymerase chain reaction (qPCR), Immunohistochemistry, Immunofluorescence and enzyme linked immunosorbent assay (ELISA) in inactive and active RA patients and healthy controls. The effect of KIAA1199 expression on FLSs proliferation, angiogenesis and related pathway were analyzed by MTT, cell migration, tube formation, chorioallantoic membrane (CAM) assay, qPCR and western-blotting after KIAA1199 knockdown and over-expression.

Results: The verification results show the up-regulation of KIAA1199 in RA patients at mRNA and protein level as compared to that in healthy controls. ELISA and receiver operator characteristic (ROC) analysis shows that KIAA1199 concentration in serum, synovial fluid and synovial tissues could be used as dependable biomarkers for the diagnosis of active RA, provided an area under roc curve (AUC) of 0.83, 0.92 and 0.92 . Sensitivity and specificity, which were determined by cut-off points, reached $72 \% 84 \%$ and $80 \%$ in sensitivity and $80 \%, 93.3 \%, 93.3 \%$ in specificity, respectively. Moreover, KIAA1199 also enhance the proliferation and angiogenesis of synovial membrane, and KIAA1199/ PLXNB3/ SEMA5A/CTGF axis may be a newly found pathway enhancing cell proliferation and angiogenesis.

Conclusion: KIAA1199 may be a potential diagnostic biomarker of RA related to angiogenesis.
\end{abstract}

\section{Introduction}

Rheumatoid arthritis (RA) is a chronic inflammatory disease characterized by progressive joint damage. The pathogenesis of RA is complex and thought to be mediated by various mechanisms. Early events in RA disease progression are defined by hyperplasia of the synovial membrane, influx of leukocytes and inflammatory cells. Activated fibroblast-like synoviocytes (FLSs) in the lining layer of the synovial membrane are among the dominant cell types involved in pannus formation, and pannus is a key player in joint destruction [1,2]. Angiogenesis is now recognized as a key event in the formation and maintenance of the pannus in RA [3,4].

\footnotetext{
*Correspondence: wangjiangmin1968@126.com; wz_wjg@163.com ${ }^{\dagger}$ Equal contributors

${ }^{3}$ Department of Rheumatology, Jiamusi Central Hospital, Jiamusi, China ${ }^{1}$ Department of Biochemistry, School of Basic Medical Sciences, Wenzhou Medical University, Wenzhou, China

Full list of author information is available at the end of the article
}

KIAA1199 gene is a member of the large transmembrane protein of the KIAA family with more than 1000 amino acids [5] discovered about 10 years ago. Human KIAA1199 gene is located on chromosome 15q25.1 segment, which encodes a $150 \mathrm{kDa}$ protein originally described as an inner ear protein [6]. KIAA1199 was found to have a G8 domain [7] and two GG domains [8]. Although the basic function of KIAA1199 remains unknown, an inverse correlation between the expression level of KIAA1199 and disease stage/5-year survival rate suggests that KIAA1199 may be associated with cancer progression [9]. It was also demonstrated that KIAA1199 was over-expressed in excessively proliferated cancer tissues, including those from gastric cancer [9], breast cancer [10-12] and colon cancer [13-18]. In addition, our previous proteomic study on FLSs derived from the synovial membrane also found that KIAA1199 expression in RA patients was significantly higher than in healthy 
controls [19], but the biological function and mechanism of action of KIAA1199 in RA remain unknown.

The aim of the present study was to verify the overexpression of KIAA1199 mRNA and protein in the serum, synovial fluid and synovial tissues obtained from patients with active and inactive RA and healthy controls, explore the effect of KIAA1199 on FLSs proliferation and angiogenesis by MTT, cell migration, tube formation and chorioallantoic membrane (CAM) assay after KIAA1199 knockdown and over-expression.

\section{Methods}

\section{Patients and primary culture of FLS cells}

The serum was obtained from 44 RA patients, 15 osteoarthritis (OA) patients, 15 ankylosing spondylitis (AS) patients and 15 normal subjects. Knee synovial fluids and synovial tissues were from 44 RA patients undergoing synovectomy or joint replacement surgery and 15 normal subjects undergoing high-level amputations in Shanghai Changhai Hospital and Shanghai Guanghua Hospital (Shanghai, China). RA patients were further categorized as a group with active RA $(\mathrm{n}=25)$ and a group with inactive RA $(n=19)$ depending on the elevation of disease activity score in 28 joints (DAS28) (inactive RA: DAS28 < 3.2; active RA: DAS28 > 3.2); DAS28 score correlates closely with clinical parameters of RA disease activity [20]. Patients fulfilled the 1987 American College of Rheumatology criteria for the diagnosis of RA [21]. The clinical data of the patients are shown in Table 1. Serum and synovial fluid were stored at $-80^{\circ} \mathrm{C}$ immediately after centrifugation at $12,000 \mathrm{rpm}$. One part of synovial tissues was stored at $-80^{\circ} \mathrm{C}$, another part was isolated enzymatically according to the method previously described [19]. All FLSs of passages three to five were used for the experiment. This study was approved by Shanghai Changhai Hospital ethics committee (CHEC2013-194), with informed consent from all the participants concerned.

\section{RNA preparation and quantitative real-time PCR analysis}

Total RNA was extracted from the synovial tissues or FLS cells by Trizol reagent (Invitrogen, Carlsbad, CA, USA), precipitated with isopropanol and dissolved in DEPC-treated distilled water. The concentration of total RNA was determined by Eppendorf BioPhotometer Plus (Hamburg, Germany). Total RNA $(2 \mu \mathrm{g})$ was then treated with RNase-free DNase (Invitrogen, Carlsbad, CA, USA) before the first-strand cDNA was generated using the random hexamer primer provided in the first-strand cDNA synthesis kit (MBI Fermantas, Vilnius, Lithuania). Specific amplification was performed using the primers of KIAA1199 genes (forward primer: 5' TGC TGC CCG GGT ATT CAA AT 3' and reverse primer: 5'CGT CCA CTC CAC GTC TTG AA 3'), plexnB3 genes (forward primer: 5' ACC CAG GTC AAG GAG AAG GT 3' and reverse primer: 5' GTC TTC GTC CGA TAG GGT CA 3'), sema5A genes (forward primer: 5' GCT CCT TCC ACA AGA AGT GC 3' and reverse primer: 5' CAA GCT GCT TCC AAG AAT CC 3'), ctgf genes (forward primer: 5' TGG AGT TCA AGT GCC CTG AC 3 ' and reverse primer: 5' GTA ATG GCA GGC ACA GGT CT 3') and $\beta$ actin (forward primer: 5'ATGG TGG GTA TGG GTC AGA AG 3' and reverse primer: 5'TGG CTG GGG TGT TGA AGG TC $3^{\prime}$ ) used as an internal control for determining the cell number and metabolic status. Quantitative real-time PCR (ABI7300, Applied Bio-systems, Carlsbad, CA, USA) was done with SsoFast EvaGreen supermix PCR kit (Bio-Rad, Hercules, CA, USA). A total of 40 cycles

Table 1 Demographic characteristics of patients and normal subjects

\begin{tabular}{|c|c|c|c|c|c|}
\hline & Normal & $\begin{array}{l}\text { Inactive rheumatoid } \\
\text { arthritis (disease activity } \\
\text { score in } 28 \text { joints }<3.2 \text { ) }\end{array}$ & $\begin{array}{l}\text { Active rheumatoid } \\
\text { arthritis (disease activity } \\
\text { score in } 28 \text { joints }>3.2 \text { ) }\end{array}$ & Osteoarthritis & AS \\
\hline Number & 15 & 19 & 25 & 15 & 15 \\
\hline Age, years & $47.2 \pm 9.91$ & $53.64 \pm 6.32$ & $57.56 \pm 9.75$ & $54.65 \pm 8.43$ & $38.75 \pm 9.34$ \\
\hline Male/female, $\mathbf{n}$ & $6 / 9$ & $8 / 11$ & $10 / 15$ & $7 / 8$ & $8 / 7$ \\
\hline Serum C-reactive protein, $\mathrm{mg} / \mathrm{dl}$ & $0.19 \pm 0.09$ & $0.20 \pm 0.07$ & $1.37 \pm 0.89$ & $0.18 \pm 0.04$ & $0.38 \pm 0.17$ \\
\hline Disease activity in 28 joints, score & $0.94 \pm 0.41$ & $2.57 \pm 1.35$ & $5.38 \pm 3.93$ & NA & NA \\
\hline Erythrocyte sedimentation rate, $\mathrm{mm} / \mathrm{h}$ & $9.83 \pm 3.06$ & $28.35 \pm 13.41$ & $47.36 \pm 29.41$ & $10.57 \pm 2.44$ & $36.9 \pm 13.52$ \\
\hline Duration of disease, years & NA & $4.1 \pm 2.7$ & $8.3 \pm 4.3$ & $6.4 \pm 2.9$ & $10.6 \pm 5.8$ \\
\hline $\begin{array}{l}\text { Bath ankylosing spondylitis disease } \\
\text { activity index, score }\end{array}$ & NA & NA & NA & NA & 7. $85 \pm 5.24$ \\
\hline $\begin{array}{l}\text { Non-steroidal anti-inflammatory drug } \\
\text { usage, } \%\end{array}$ & NA & 78.3 & 81.3 & NA & 46.6 \\
\hline $\begin{array}{l}\text { Disease-modifying anti-rheumatic drug } \\
\text { usage, } \%\end{array}$ & NA & 63.5 & 65.1 & NA & 38.9 \\
\hline
\end{tabular}

Values are expressed as mean \pm SD unless stated otherwise. NA, not applicable. 
of PCR was performed for $15 \mathrm{~s}$ at $95^{\circ} \mathrm{C}$, and $60 \mathrm{~s}$ at $60^{\circ} \mathrm{C}$. The relative expression of each target gene compared with $\beta$-actin was calculated using the $2^{-\Delta \Delta C t}$. All reactions were conducted in triplicate.

\section{Immunohistochemical analysis}

Synovial tissues were fixed overnight in $4 \%$ paraformaldehyde, embedded in paraffin, and sectioned in 5- to 8- $\mu \mathrm{m}$ intervals. In brief, sections on slides were de-paraffinized, re-hydrated, antigens unmasked by incubating in target retrieval solution at $95^{\circ} \mathrm{C}$ for 30 minutes, permeabilized in $0.1 \%$ Triton-X100 for 5 minutes, blocked with $10 \%$ chicken serum in TBST for 45 minutes, and incubated with KIAA1199 monoclonal antibody (sc-164775, Santa Cruz, CA, USA) at $1 / 20$ at $37^{\circ} \mathrm{C}$ overnight. Subsequently, a peroxidase 3,3-diaminobenzidine (DAB) detection system (SK6333-2; Sangon Biotech, Shanghai, China) was applied according to the manufacturer's instructions. The sections were observed under a fluorescence microscope.

\section{Immunofluorescence microscopy}

FLS cells grown on glass coverslips were washed with PBS, fixed at room temperature with $4 \%$ paraformaldehyde (20 minutes ), permeabilized with $0.5 \%$ Triton-X 100 (10 minutes), and blocked with $10 \%$ normal goat serum (30 minutes). They were then incubated with KIAA1199 monoclonal antibody (sc-164775, Santa Cruz, CA, USA) and CTGF (ab6992, abcam) overnight at $4^{\circ} \mathrm{C}$, and then with secondary antibodies (rabbit anti-goat IgG Dylight ${ }^{\mathrm{m}}$ 549 conjugated and donkey anti-rabbit IgG Alexa Fluor 488 conjugated , catalog number: BM8505 and BMJ8106, Bio Mart, New Delhi, India) for 45 minutes at $37^{\circ} \mathrm{C}$. The cells were covered with 4',6-diamidino-2-phenylindole (DAPI)-Vectashield mounting medium (236276, Roche, Switzerland), and images were captured on an epifluorescence microscope (Leica, Wetzlar, Hessen, Germany) equipped with Leica Application Suite V3.3.0 software.

\section{Enzyme-linked immunosorbent assay for human KIAA1199} KIAA1199-specific antigens were detected in serum, synovial fluid and synovial tissues extraction samples from patients with active RA, inactive RA, OA and AS, and healthy subjects. The homogenate was centrifuged in a micro-centrifuge for 5 minutes at $3,000 \mathrm{~g}$, and $100 \mu \mathrm{l}$ diluted supernatant (1:50 with incubation buffer) was incubated at room temperature in a microtiter plate coated with KIAA1199 monoclonal antibody (sc-164775, Santa Cruz, CA, USA). After incubation, washing and addition of a detection antibody coupled to horseradish peroxidase, the substrate was added and incubated, followed by addition of a stop solution. The absorption rate was determined at an optical density of $450 \mathrm{~nm}$. All reactions were conducted in triplicate.

\section{KIAA1199 knockdown and over-expression}

KIAA1199 knock-down experiments were performed by transfecting KIAA1199-specific siRNA (100nM). The siRNA sequence targeting KIAA1199 was 5' -AAA CAU UGA AAU AUU CGC CAU GCU C- 3' and 5' -UUG ACA AGG AGG CCA AGA CAG UGG U- 3'. Scrambled siRNA was 5'-UUU UCG CUG CGC CAA CCU CTT-3' and 5' -AUA AGG GAA CGU GAG CGC GTT 3'. KIAA1199 over-expression experiments were performed by transfecting pcDNA3.2DEST-cloned KIAA1199 open reading frame $(100 \mathrm{nM})$, which was supplied freely by Dr Marra (Institute of Molecular Cancer Research, University of Zurich, Zurich, Switzerland) [15].

\section{Western blotting analysis}

An equal amount of protein extract (50 $\mu \mathrm{g}$ per lane) was separated by SDS-PAGE of $10 \%$ polyacrylamide, and electrotransferred onto polyvinylidene fluoride (PVDF) membranes. The membranes were incubated for $1 \mathrm{~h}$ with a blocking solution containing $0.1 \%$ Triton X-100, $5 \%$ nonfat milk in PBS. The KIAA1199 monoclonal antibody (Abnova, H00057214-M01), PLXNB3 (Santa Cruz, sc-46240), SEMA5A (sc-67953, Santa Cruz, CA, USA), CTGF (R\&D Systems, AF660, Minneapolis, MN, USA) were then added. The membranes were incubated overnight at $4^{\circ} \mathrm{C}$, and then with the appropriate horseradish peroxidase-conjugated secondary antibody at room temperature for $1 \mathrm{~h}$. The filter was then incubated with the substrate and exposed to radiographic film. All reactions were conducted in triplicate.

\section{Cell proliferation assay}

Cell proliferative activities were examined using FLSs. Cells were seeded onto 96-well plates $\left(1 \times 10^{4}\right.$ cells/well $)$ for $24 \mathrm{~h}$ and treated with a fresh culture medium containing various concentrations of KIAA1199 siRNA duplex $(50,100,200 \mathrm{nM})$ or scrambled siRNA for $72 \mathrm{~h}$ at $37^{\circ} \mathrm{C}$. The proliferative capacity of FLSs was determined by the MTT-based cell proliferation and viability assay system according to the manufacturer's instructions. The results showed that the viability of KIAA1199 siRNAtreated FLSs decreased significantly in a dose-dependent manner (data not shown), and 100-nM KIAA1199 siRNA duplex was used in this study. FLS cells were seeded onto 96-well plates for $24 \mathrm{~h}$ and transfected with scrambled siRNA, KIAA1199 siRNA duplex (100nM), pcDNA3.2DEST and pcDNA3.2DEST-KIAA1199 (100 $\mathrm{nM})$ for $24 \mathrm{~h}, 48 \mathrm{~h}, 72 \mathrm{~h}$ and $96 \mathrm{~h}$ at $37^{\circ} \mathrm{C}$ by Lipofectamine 2000 (Invitrogen, Carlsbad, CA, USA) according to the manufacturer's protocol. The proliferative capacity of FLSs was determined by the MTT-based cell proliferation and viability assay system. The differences in absorbance were compared in vector control and KIAA1199-transfected cells. The assay was performed in triplicate. 


\section{Cell migration and endothelial tube formation assays}

Transwell motility assays were performed by $6.5 \mathrm{~mm}$ Transwell $^{\bullet}$ with $8.0-\mu \mathrm{m}$ pore polycarbonate membrane filters (Corning Corp, Corning, NY, USA). Human umbilical vein endothelial cells (HUVEC) transfected with scrambled siRNA, KIAA1199 siRNA duplex (100 nM), pcDNA3.2DEST and pcDNA3.2DEST-KIAA1199 (100 nM) were cultured to $85 \%$ to $95 \%$ confluence, subjected to serum starvation for $24 \mathrm{~h}$ before cell migration assay, dissociated by incubation with trypsin-EDTA, washed twice with PBS, and counted using a hemocytometer. Then $600 \mu \mathrm{l}$ medium was added to each lower chamber of the 24-well transwell for $24 \mathrm{~h}$ at $37^{\circ} \mathrm{C}$. Inserts (the upper chambers) were then placed in the wells. Cells $\left(1 \times 10^{5}\right.$ in $300 \mu$ l serum-free media) were added to each upper chamber and incubated for $6 \mathrm{~h}$. Nonmigrating cells were removed with a cotton swab. Cells that migrated to the lower phase of the upper chamber were then fixed in methanol for 30 minutes and stained with crystal violet for 3 minutes at room temperature. Excess stain was removed with distilled water, and the chambers were air-dried. Pictures were taken under the microscope and the cell number was quantified by software Image-Pro. The assay was performed in triplicate.

The tube formation assay was performed as follows: 24well plates were pre-coated with Matrigel and incubated at $37^{\circ} \mathrm{C}$ to promote gelling. HUVEC were added to each well transfected with scrambled siRNA, KIAA1199 siRNA duplex (100 nM), pcDNA3.2DEST and pcDNA3.2DESTKIAA1199 (100 nM). After $6 \mathrm{~h}$ incubation, the plates were fixed with $4 \%$ paraformaldehyde and a blinded observer assessed the morphology of the tubes. Tube-like structures were quantified by counting the number of intersections between branches of the endothelial cell networks in the whole field. The assay was performed in triplicate.

\section{Chorioallantoic membrane assay}

Angiogenic activity of KIAA1199 was assayed on CAM as described by Takigawa et al. [22]. Embryonic CAM were treated on day 7 with scrambled siRNA, KIAA1199 siRNA duplex (100 nM), pcDNA3.2DEST and pcDNA3.2DESTKIAA1199 (100 nM) absorbed on sterile Whatman GB/B glass fiber filter disks (6 $\mathrm{mm}$ in diameter; Reeve-Angel, Clifton, NJ, USA). The disks prepared with $20 \mu \mathrm{l}$ of factor were placed upside down on windows that had been made in the eggshells on day 7 of incubation. The embryos were examined 3 days later under a stereomicroscope. ImageJ $2.43 \mathrm{~s}$ was used to calculate the vascular area and CAM area.

\section{Statistical analysis}

Data analysis involved estimation of the mean and SD using SPSS 17.0. The Shapiro-Wilk method and histograms were used to test whether the data were normally distributed. The Levene method was used to test homogeneity of variance. Two sets of data that met the normal distribution and homogeneity of variance were analyzed by independent samples $t$-test. The Kruskal-Wallis and Mann-Whitney non-parametric tests were used to compare interassay differences in data that did not meet the normal distribution or the homogeneity of variance. Inspection level $P$-values $<0.05$ were considered statistically significant.

\section{Results}

\section{Elevated expression of KIAA1199 in RA patients}

Our previous comparative proteomic study results showed that KIAA1199 protein was 5.19 times over-expressed in RA FLSs [19]. The results of automated 2D-Nano-LCESI-MS/MS identification are shown in Figure $1 \mathrm{~A}$ and Additional file 1. In this study quantitative real-time PCR was performed to confirm whether there was a real difference between RA and normal synovial tissues in the expression of KIAA1199. Compared to healthy subjects, the KIAA1199 mRNA expression was 2.77-fold and 6.62-fold up-regulated in inactive RA and active RA patients than in healthy subjects, respectively (Figure $1 \mathrm{~B}$ ), which was consistent with the change in the previous proteomic study. The immunohistochemical analysis shows the expression of KIAA1199 was significantly higher in the vascular endothelium of synovial membrane in active RA and inactive RA than in normal synovial membrane (Figure 1C). Immunofluorescence microscopy shows that KIAA1199 and CTGF proteins were co-expressed in FLS cells, and they were either not expressed or only weakly expressed in the nucleus, and were strongly expressed in the cytoplasm and membrane of FLS cells (Figure 1D).

\section{Correlation between KIAA1199 and disease activity}

Knowing that DAS28 score is an indicator for measuring clinical disease activity, RA patients were divided into an active or inactive group according to DAS28 score (inactive RA: DAS $28<3.2$; active RA: DAS28 > 3.2). The abundance levels of KIAA1199 in the serum, synovial fluid and synovial tissues of patients with inactive and active RA and healthy subjects were determined by ELISA. KIAA1199 protein was expressed in human serum, synovial fluid and synovial tissue, while the expression of KIAA1199 protein was increased in the RA group compared to the healthy group $(P<0.05)$. There were no significant differences in KIAA1199 in serum among patients with OA or AS, or healthy controls, and the concentration of KIAA1199 in RA was different from that of OA and AS. Therefore the concentration of KIAA1199 can be used as a biomarker of RA. The levels of KIAA1199 in serum, synovial fluid and synovial tissue were significantly elevated in patients with active RA as compared to those with inactive RA. Interestingly, the number of samples that were KIAA1199-positive was significantly greater in the active RA than that in the 


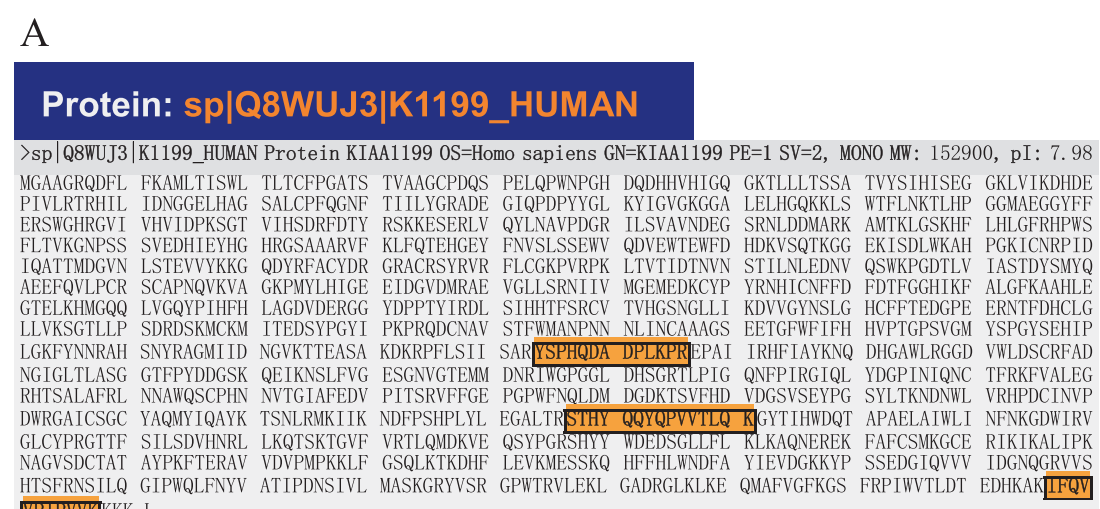
VPIPVVKKKK L

B

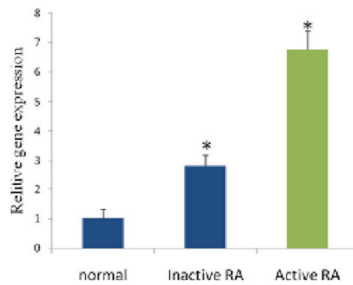

normal moctive Pa Active RA

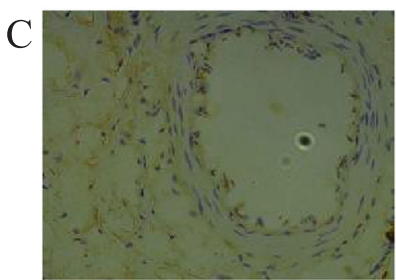

Normal

D

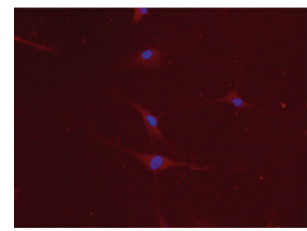

KIAA1199+DAPI

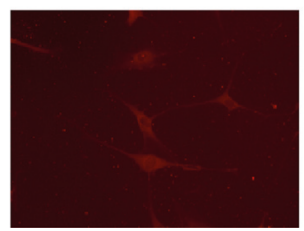

KIAA1199



CTGF+DAPI



Inactive $\mathrm{RA}$

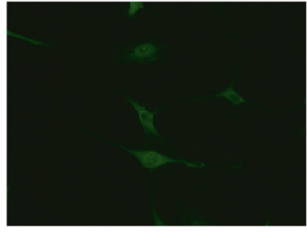

CTGF

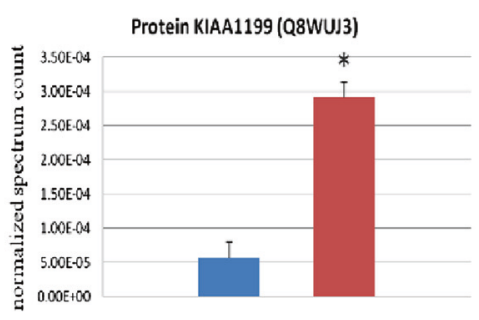

normal RA

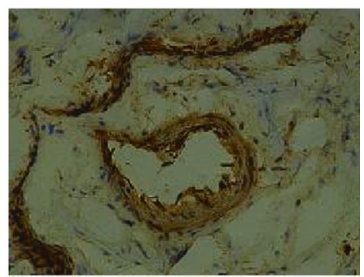

Active RA

Figure 1 Identification and verification of KIAA1199 expression in rheumatoid arthritis (RA) patients and normal subjects. (A) Identification the overexpression of KIAA1 199 in RA fibroblast-like synoviocyte (FLS) cells than in normal FLS cells from synovial membrane by automated 2D-Nano-LC-ESI-MS/ MS. The left panel shows amino acid coverage of the identified KIAA1199 sequence; yellow, appraisal sequence of amino acids. The right panel is the normalized spectrum count in RA and normal subjects; ${ }^{*} P<0.001$. (B) Verification of the expression of KIAA 1199 was performed in synovial tissues from 19 patients with inactive RA, 25 with active RA and 15 normal subjects by qPCR. Results were normalized for the amount of $\beta$-actin as internal control. Experiments were performed at least in triplicates; bars represents mean \pm SD; ${ }^{*} P<0.05$ compared with normal. (C) Immunohistochemical staining of KIAA1199 was performed on synovial tissues from patients with inactive or active RA and normal subjects. The KIAA1199 protein was over-expressed in vascular endothelium in active and inactive RA synovial membrane compared to normal synovial membrane. (D) Immunofluorescence microscopy of KIAA1199 and CTGF was performed in FLS cells from RA patients. Result shows KIAA1199 and CTGF were co-expressed in FLS cells, were either not expressed or only very weakly expressed in the nucleus, and were strongly expressed in the cytoplasm and membrane of FLS cells.

inactive RA group (18 versus 11 in the serum, 21 versus 9 in the synovial fluid, and 20 versus 9 in the synovial tissues).These data suggest that the level of KIAA1199 in serum, synovial fluid and synovial tissues was correlated with the disease activity and was associated with the process of RA (Figure 2).

\section{ROC curve analysis determined the diagnostic value of KIAA1199 for RA}

In order to determine the diagnostic value of KIAA1199 for RA, we conducted a receiver operator characteristic (ROC) curve analysis of KIAA1199. The result reveals that the areas under the ROC curve for the serum, synovial fluid and synovial tissues from inactive RA were 0.74, 0.78 , and 0.79 (range 0.7 to 0.8 ), respectively, indicating medium diagnostic value. The area under the ROC curve for serum, synovial fluid and synovial tissues from active RA were $0.83,0.92$ and 0.91 , respectively, indicating high diagnostic value. Cutoff values of 90.13, 129.1 and $198.7 \mu \mathrm{g} / \mathrm{ml}$ were able to detect individuals with active RA at the sensitivity of $72 \%, 84 \%$ and $80 \%$, respectively, and specificity of $80 \%, 93.3 \%$ and $93.3 \%$, respectively (Figure 2). The results show better diagnostic value of KIAA1199 in synovial fluids and synovial tissue in RA. 
A ROC Curves of serum

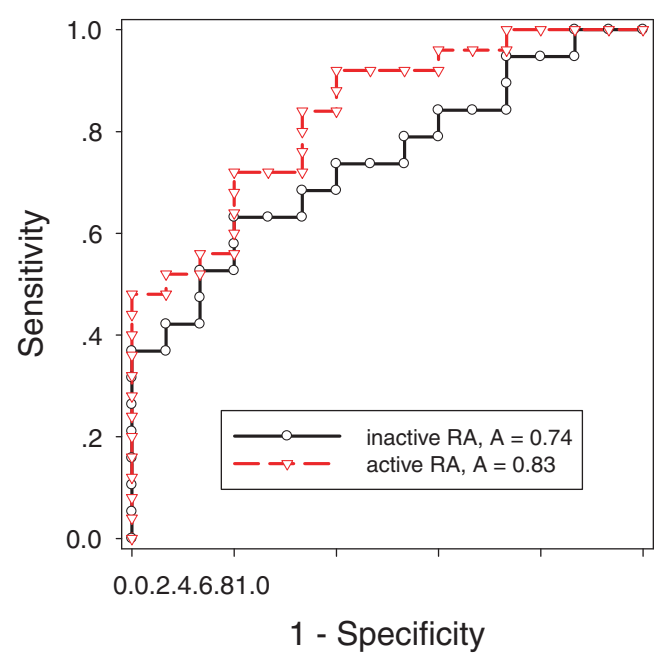

B ROC Curves of synovial fluids

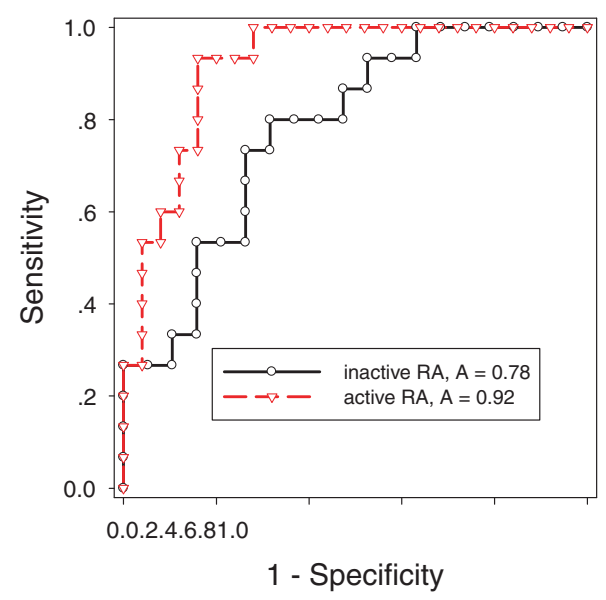

C ROC Curves of synovium

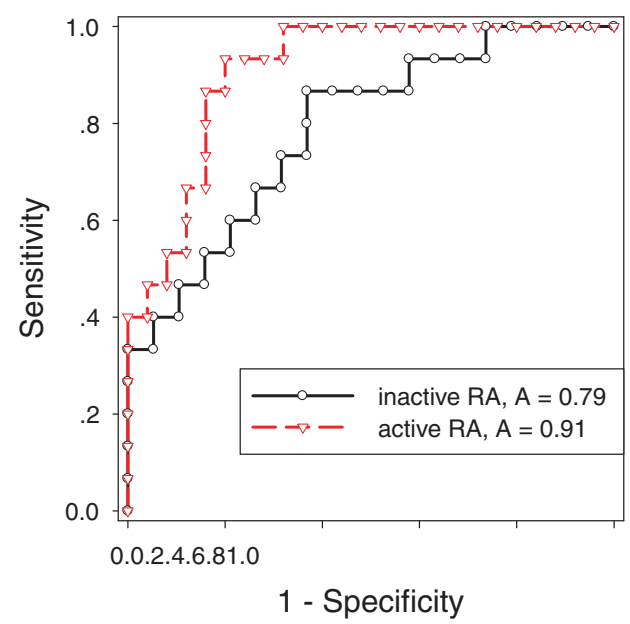

Serum

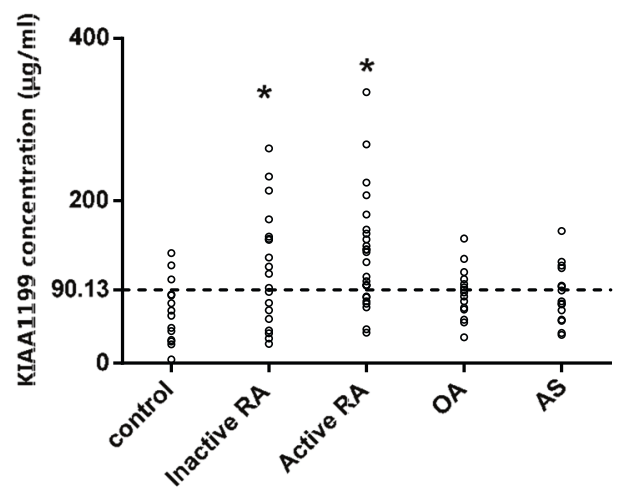

Synovial fluids



Synovium

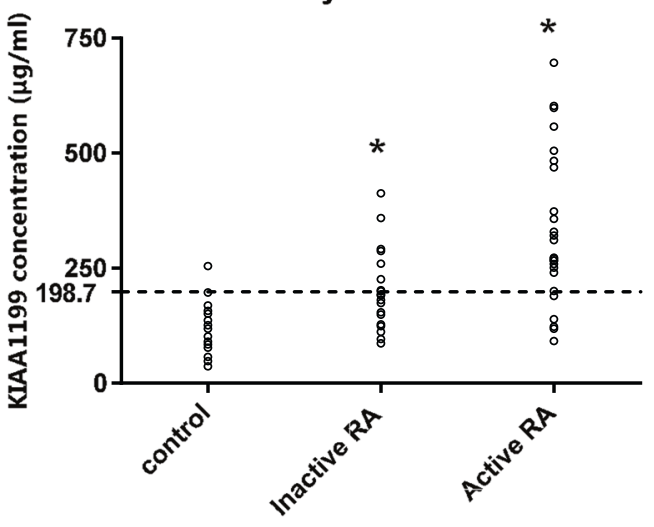

Figure 2 (See legend on next page.) 
(See figure on previous page.)

Figure 2 Diagnostic value of evaluation of KIAA1199 in rheumatoid arthritis (RA) determined by receiver operator characteristic (ROC) curve analysis. (A) ROC curve analysis of KIAA1199 in serum for diagnosis of RA. The area under the curve (AUC) for inactive RA-healthy controls (HC) and active RA-HC was 0.74 and 0.83 , respectively. A cutoff value of $90.13 \mu \mathrm{g} / \mathrm{ml}$ diagnosed active RA at the sensitivity of $72 \%$ and the specificity of $80 \%$, respectively. (B) ROC curve analysis of KIAA1199 in synovial fluids for the diagnosis of RA. The AUC for inactive RA-HC and active RA-HC was 0.78 and 0.92 , respectively. A cutoff value of $129.1 \mu \mathrm{g} / \mathrm{ml}$ diagnosed active RA at the sensitivity of $84 \%$ and the specificity of $93.3 \%$, respectively. (C) ROC curve analysis of KIAA1199 in the synovium for the diagnosis of RA. The AUC for inactive RA-HC and active RA-HC was 0.79 and 0.91 , respectively. A cutoff value of $198.7 \mathrm{\mu g} / \mathrm{ml}$ diagnosed active RA at the sensitivity of $80 \%$ and the specificity of $93.3 \%$, respectively. All experiments were performed at least in triplicates; ${ }^{*} P<0.05$ compared with control. OA, osteoarthritis; AS, ankylosing spondylitis.

\section{The effect of KIAA1199 on FLS cells proliferation}

The proliferation of synovial membrane is now recognized as a key event in RA progression. To determine the effects of KIAA1199 expression on FLS cell proliferation, the MTT assay was performed on FLS cells treated with scrambled siRNA, KIAA1199 siRNA duplex, pcDNA3.2DEST and pcDNA3.2DEST-KIAA1199 for $24 \mathrm{~h}, 48 \mathrm{~h}, 72 \mathrm{~h}$ and $96 \mathrm{~h}$ at $37^{\circ} \mathrm{C}$. The MTT results showed that the viability of FLSs treated with KIAA1199 siRNA decreased significantly after KIAA1199 knock-down and increased after KIAA1199 over-expression; significant differences were observed between every time point examined, indicating a linear change in proliferation with treatment time, $P<0.05$. These data support the assumption that KIAA1199 may be an inducer of FLSs proliferation (Figure 3).

\section{The effect of KIAA1199 on angiogenesis}

Angiogenesis is now recognized as a key event in the formation and maintenance of the pannus in RA. To further evaluate the effects of KIAA1199 expression on cell angiogenesis, the transwell and tube formation assays were performed on HUVEC cells treated with scrambled siRNA, KIAA1199 siRNA duplex, pcDNA3.2DEST and

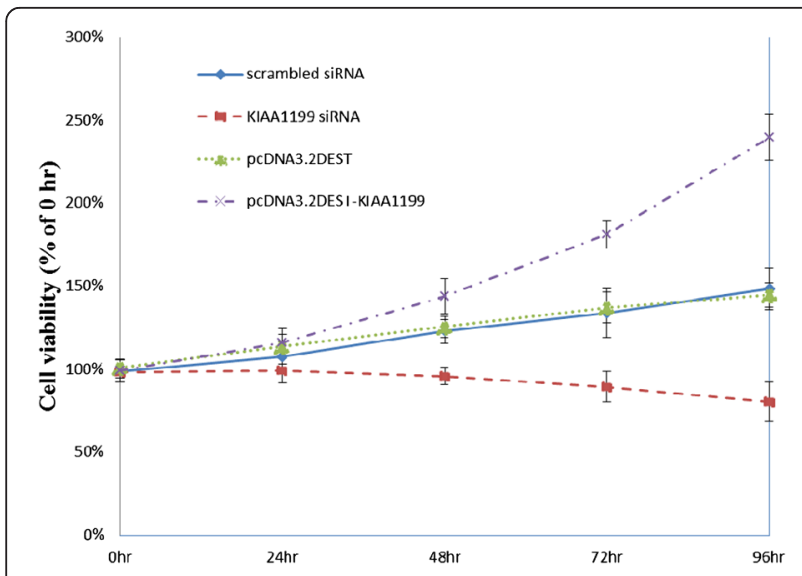

Figure 3 The effect of KIAA1199 expression on fibroblast-like synoviocyte (FLS) cell proliferation detected by MTT assay. FLS cells isolated from synovial tissue were transfected with scrambled siRNA, KIAA1199 siRNA duplex, pcDNA3.2DEST and pcDNA3.2DESTKIAA1 199 for $24 \mathrm{~h}, 48 \mathrm{~h}, 72 \mathrm{~h}$ and $96 \mathrm{~h}$ at $37^{\circ} \mathrm{C}$. Cell viability was determined by MTT. All experiments were performed at least in triplicates.
pcDNA3.2DEST-KIAA1199 for $6 \mathrm{~h}$ at $37^{\circ} \mathrm{C}$. The transwell assay result shows KIAA1199 knock-down reduced the migration ability and KIAA1199 over-expression increased the migration ability of HUVEC cells, $P<0.05$ (Figure 4A). The tube formation experiment shows a similar result to the transwell assay, $P<0.05$ (Figure $4 \mathrm{~B}$ ). The above in vitro findings allowed us to investigate the angiogenic activity of KIAA1199 in vivo. First, we carried out a CAM assay. After 3 days treatment of scrambled siRNA, KIAA1199 siRNA duplex, pcDNA3.2DEST and pcDNA3.2DESTKIAA1199 on CAM of chick embryos, the formation of small vessels decreased in KIAA1199 knock-down and increased in the KIAA1199 over-expression experiment, $P<0.05$, suggesting that KIAA1199 is a requirement for angiogenesis (Figure 4C).

\section{Effect of the KIAA1199/PLXNB3/ SEMA5A/CTGF axis on angiogenesis}

To clarify the pathway of KIAA1199 regulation of angiogenesis in FLS cells in RA, hypothetical-pathway-related proteins were determined by qPCR and western blotting in triplicate. We observed an decrease of $77.0 \%, 70.3 \%$, $51.7 \%$, and $64.9 \%$ in RNA levels and a decrease of $76.5 \%$, $54.6 \%, 47.0 \%$, and $30.7 \%$ in protein levels of KIAA 1199 , PLXNB3, SEMA5A and CTGF after KIAA1199 knockdown, and an increase of $182.6 \%, 135.5 \%, 110.3 \%$, and $132.2 \%$ in RNA levels and an increase of $77.5 \%, 40.7 \%$, $75.4 \%$, and $112.0 \%$ in protein levels of KIAA1199, PLXNB3, SEMA5A and CTGF after KIAA1199 overexpression, respectively. This is to say, the expression difference of PLXNB3, SEMA5A and CTGF were proportional to the expression of KIAA1199 in mRNA and protein levels (Figure 5A and $\mathrm{B}$ ).

\section{Discussion}

A hallmark of RA is the pseudo-tumoral expansion of FLSs, which induces the pannus formation and erodes the cartilage and bone. Many observations [23] have prompted speculation that joint FLSs in RA evolve genetically to form a locally invasive and metaplastic tissue. So cytokineenhanced FLS proliferation in RA would be a potential biomarker and has therefore been proposed as a therapeutic target by knocking down its expression. 


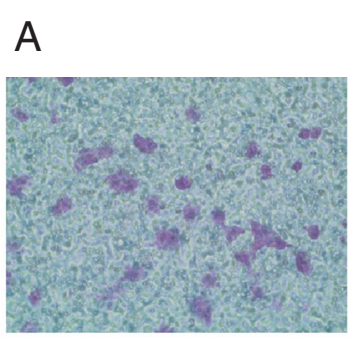

scrambled siRNA

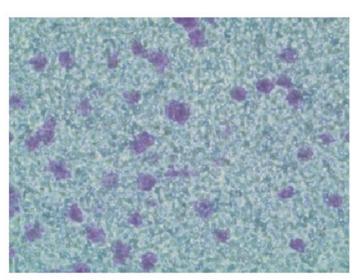

pcDNA3.2DEST

B

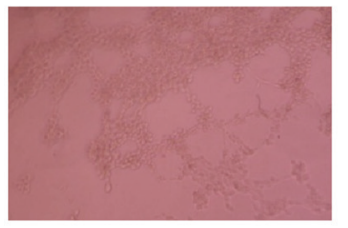

scrambled siRNA



pcDNA3.2DEST

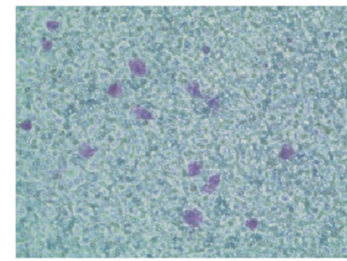

KIAA1199 siRNA

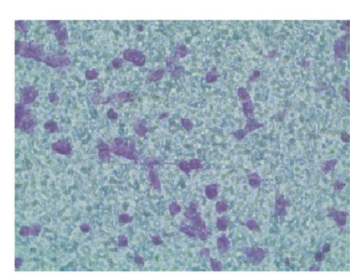

KIAA1199

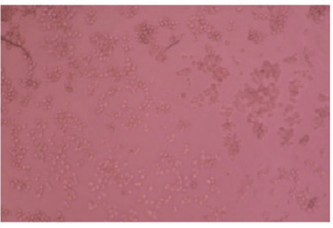

KIAA1199 siRNA

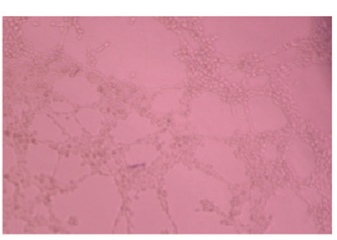

KIAA1199
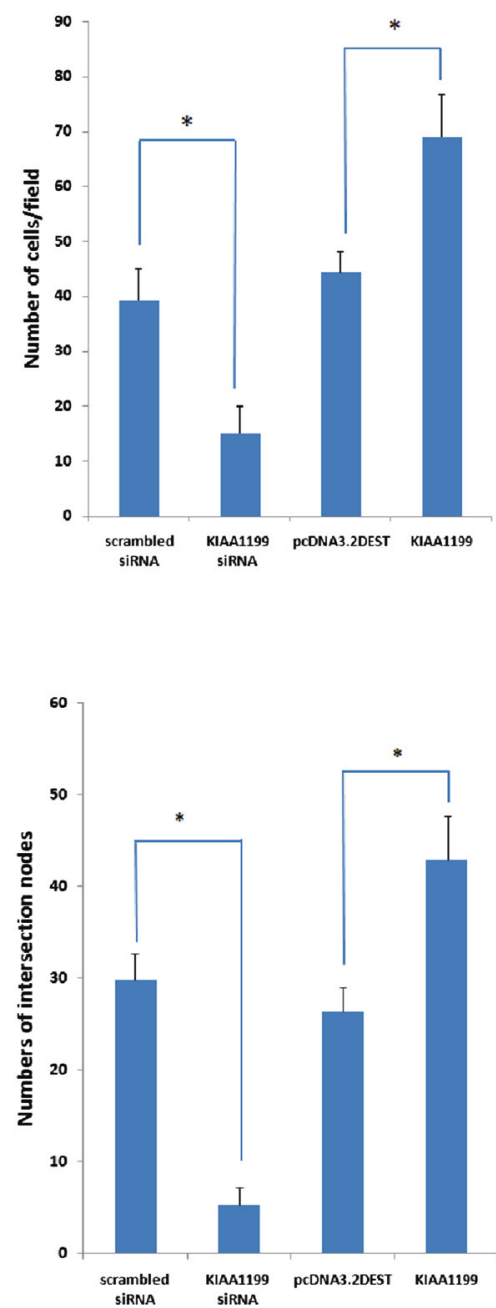

C
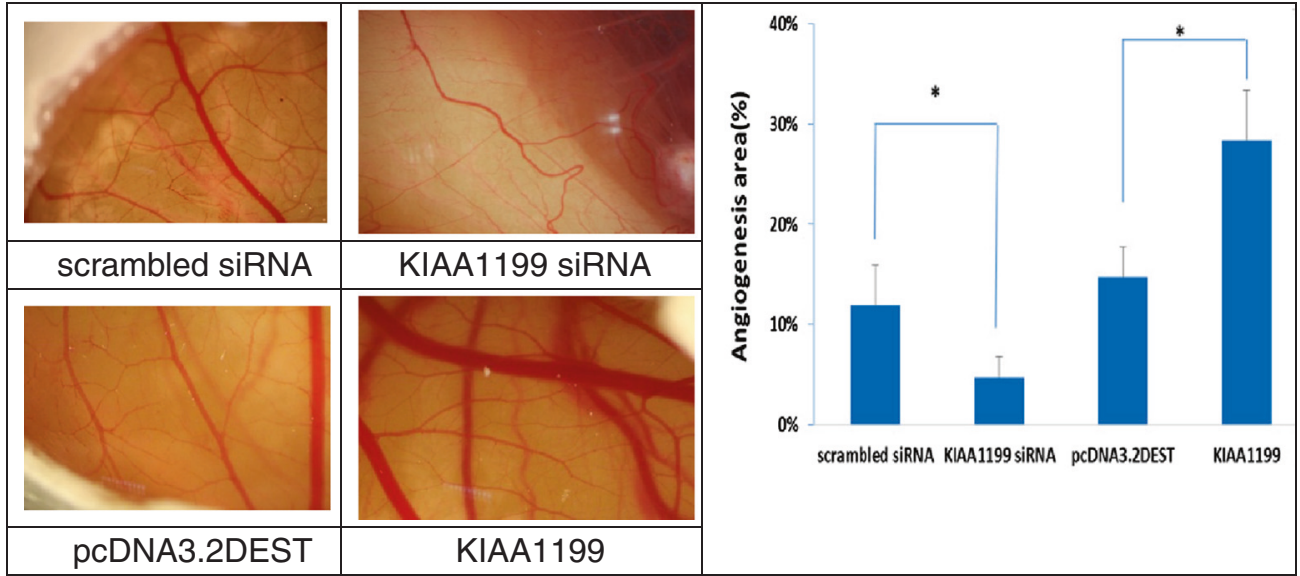

Figure 4 (See legend on next page.) 
(See figure on previous page.)

Figure 4 Effect of KIAA1199 expression on angiogenesis. (A) Cell migration assay. Human umbilical vein endothelial cells (HUVEC) transfected with scrambled siRNA, KIAA1199 siRNA duplex, pcDNA3.2DEST and pcDNA3.2DEST-KIAA1199 were plated onto the top of the transwell, allowed to migrate for $4 \mathrm{~h}$, then rinsed, fixed, stained, and counted. Left panel: HUVEC on the undersurface of a filter, magnification $\times 40$; right panel: number of HUVEC per field. (B) Tube formation assay: 24-well plates were pre-coated with Matrigel (diluted 1:2 in $\mathrm{H} 2 \mathrm{O}$ ) and incubated at $37^{\circ} \mathrm{C}$ to promote gelling. HUVEC were seeded on Matrigel-coated plates, treated with scrambled siRNA, KIAA1199 siRNA duplex, PCDNA3.2DEST and pCDNA3.2DEST-KIAA1199 for 6-8 h, tube formation observed using a phase-contrast inverted microscope, and photographs were taken from each well. The number of intersections between branches of assembled endothelial cell networks was counted in the whole field. (C). Chorioallantoic membrane (CAM) assay, embryonic CAM was treated on day 7 with scrambled siRNA, KIAA1199 siRNA duplex, pcDNA3.2DEST and pCDNA3.2DEST-KIAA1199. After incubation for 3 days CAM was examined under a stereomicroscope, and photographs taken (left panel). ImageJ $2.43 \mathrm{~s}$ was used to calculate the vascular and CAM areas. Bar graph: ratio of vascular area to CAM area (right panel). All experiments were performed at least in triplicates, values are presented as mean $\pm S D ;{ }^{*} P<0.05$ compared with control (*significant differences).

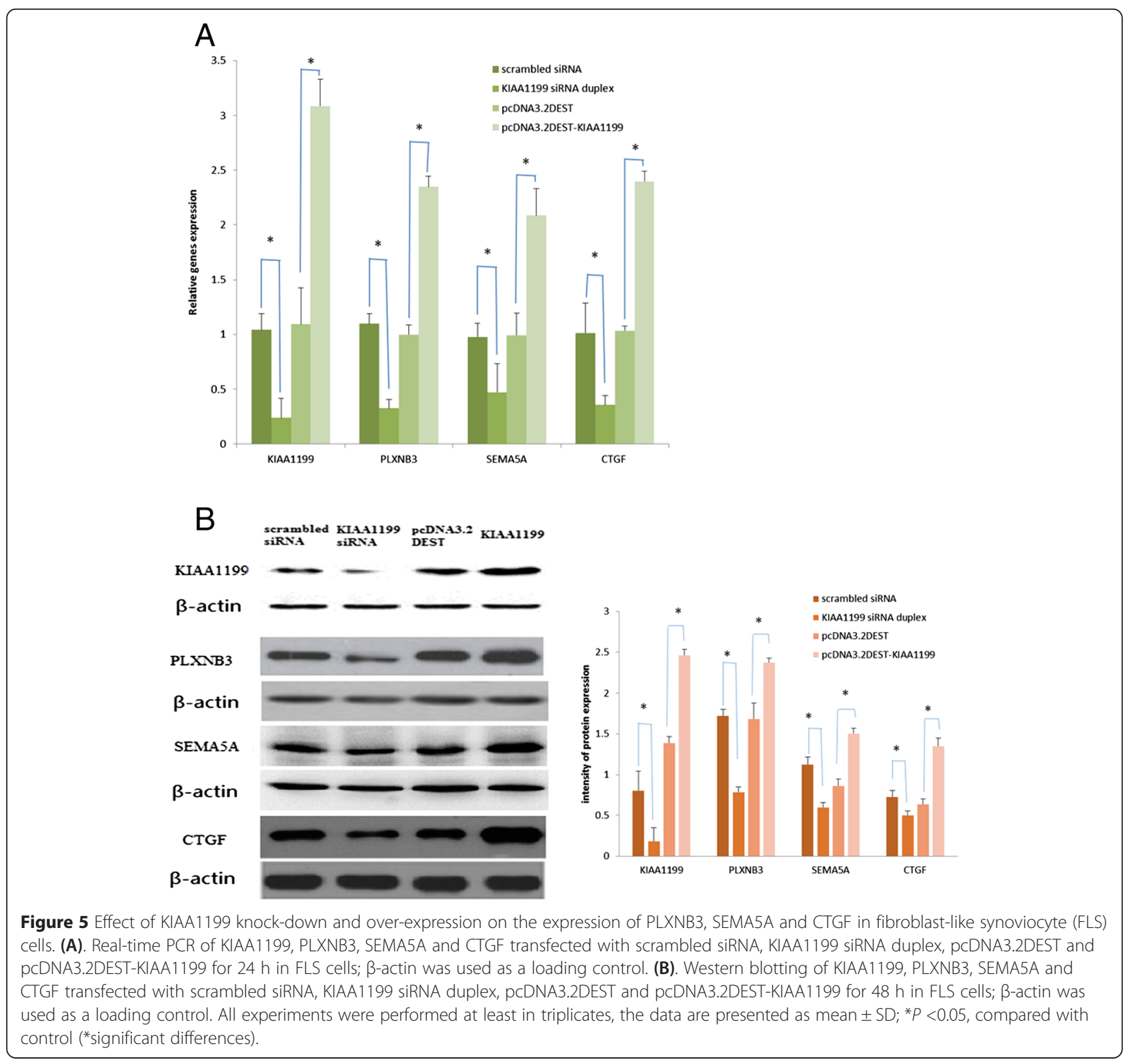


Our previous comparative proteomic study showed that KIAA1199 was 5.19 times over-expressed in RA FLS cells as identified by automated 2D-Nano-LC-ESIMS/MS [19]. This finding is supported by Yoshida's result showing that the level of KIAA1199 expression in non-inflamed synovial tissues was lower than in that of rheumatoid synovial tissues as shown by real time PCR and immunoblotting $(\mathrm{n}=3)$ [24]. However, this has not been confirmed in a large set of samples, neither with regard to RNA or protein levels, nor with regard to gene function. In this study, we evaluated KIAA1199 expression by quantitative RT-PCR and ELISA in 44 patients with RA (19 with inactive and 25 with active RA) and 15 healthy controls. The data were analyzed to determine the clinical significance of KIAA1199 levels in RA. It was found that the expression of KIAA1199 mRNA and protein were higher in the synovial tissues of RA patients than healthy subjects; ELISA also showed that the levels of KIAA1199 protein expression were higher in the serum, synovial fluid and synovial tissue from RA patients than healthy subjects, suggesting that the overexpression of KIAA1199 plays an important role in the pathogenesis of RA. These data not only indicate that the level of KIAA1199 expression is higher in RA but suggest positive correlation between KIAA1199 expression and the DAS28 score, an RA index correlated closely with clinical parameters of RA disease activity. ROC curve analysis indicated high diagnostic value of KIAA1199 in active RA. These data also imply that KIAA1199 may be a potential diagnostic biomarker of $\mathrm{RA}$, but its role in RA procession remains elusive.

Yoshida et al. carried out further research on the relationship between KIAA1199 and hyaluronan metabolism, a very important polysaccharide in synovial fluid for minimizing friction between the bones. They found that the cleavage of N-terminal 30 amino acids occurs in functionally matured KIAA1199, resulting in altered intracellular trafficking of the molecule and loss of cellular hyaluronic acid (HA) depolymerization. This suggests that the N-terminal portion of KIAA1199 functions as a cleavable signal sequence required for proper KIAA1199 translocation and KIAA1199-mediated HA depolymerization. Notably, the secreted mature form of KIAA1199 showed no HA degrading activity, together supporting the idea that KIAA1199mediated HA depolymerization occurred through rapid vesicle endocytosis. These results show that KIAA1199 protein promoted the degradation of $\mathrm{HA}$, which is normal in physiological tissues and fast in inflammatory and neoplastic diseases. In osteoarthritis or RA synovial fibroblasts, the enhancement of HA metabolism is associated with increased expression of KIAA1199 [19,24-26]. These data indicate that KIAA1199 plays a key role in HA catabolism as a unique hyaluronic cadherin in the dermal and OA synovial tissues.
The over-expression of KIAA1199 has been identified in many proliferative tissues, including synovial tissues in RA. Several reports [27] support the importance of the Wnt pathway activation in FLS proliferation. Wnt signaling is now well-recognized as a critical pathway in the regulation of growth and development. BirkenkampDemtroder et al. [17] report that KIAA1199 expression is markedly reduced by inactivation of the $\beta$-catenin/Tcell factor transcription complex, the pivotal mediator of Wnt signaling. Thus, they identified KIAA1199 as a novel target of the Wnt signaling, the regulation of KIAA1199 by Wnt signaling was observed as a proteinprotein interaction.

Immunohistochemical analysis in this study showed the expression of KIAA1199 was significantly higher in the vascular endothelium. Jami also revealed the involvement of KIAA1199 in breast cancer growth, motility and invasiveness by functional proteomic analysis [28]. Together these data suggest that KIAA1199 not only correlated with the proliferation of FLSs but may also be related to angiogenesis. The enhancing effects of KIAA1199 on angiogenesis were verified successfully in this study by transwell, tube formation and CAM assays after KIAA1199 knock-down and over-expression. CTGF is another overexpressed protein in RA patients identified in our previous proteomic study, which promotes the proliferation and migration of HUVEC [19]. Furthermore, the expression of CTGF was decreased or increased as KIAA1199 was knocked down or over-expressed. The question remains as to which pathway regulates the expression of CTGF by KIAA1199. Immunofluorescence microscopy of KIAA1199 and CTGF was performed in FLS cells from RA patients. The result shows that KIAA1199 and CTGF were coexpressed in FLS cells, and they were either not expressed or only very weakly expressed in the nucleus, and were strongly expressed in the cytoplasm and membrane of FLS cells. This experimental study gave us much inspiration. Nakayama found out that PLXNB3 is the only protein that can interact with KIAA1199, as identified by a yeast twohybrid system [29]. PLXNB3 belongs to the plexin family $[30,31]$ and is the receptor of SEMA5A, which is a membrane protein belonging to semaphorin (arm board protein) gene family with seven repeated platelet-response protein-1 domains [32]. Furthermore, there is a plateletresponse protein-1 binding domain in CTGF [33], reminding us of the interaction between SEMA5A and CTGF by bioinformatics analysis. The binding of PLXNB3 and SEMA5A is involved in combination axon guidance [34], cell invasive growth [35], angiogenesis [36] and the process of cell migration [37]. Interestingly the synovial membrane also showed the characteristics of invasive growth and angiogenesis in the progression of RA. In our experiment the expression of PLXNB3, SEMA5A and CTGF are in accordance with KIAA1199 knock-down and 
over-expression. So we boldly assume that the KIAA1199/ PLXNB3/SEMA5A/CTGF axis may accelerate the proliferation of FLS cells and activate the downstream angiogenic signaling pathways, leading to the formation of the pannus, and erode the cartilage and bone in the progression of RA.

\section{Conclusion}

One of the interesting findings in this study is the more abundant expression of KIAA1199 in RA, and the KIAA1199/PLXNB3/SEMA5A/CTGF axis may be a newly found pathway enhancing cell proliferation and angiogenesis. In addition, KIAA1199 seems to have clinical correlation with the DAS28 and disease activity. In summary, KIAA1199 may be a potential biomarker and therapeutic target in RA.

\section{Additional file}

Additional file 1: Figure S1. MS and MS/MS spectra of the peptide of KIAA1199. (A) MS and MS/MS spectrum of peptide STHYQQYQPWTLQK. (B) MS and MS/MS spectrum of peptide YSPHQDADPLKPR. (C) MS and MS/MS spectrum of peptide IFQWPIPWK.

\section{Abbreviations \\ AS: ankylosing spondylitis; AUC: area under the receiver operator characteristic curve; CAM: chorioallantoic membrane; DAS28: disease activity score in 28 joints; ELISA: enzyme-linked immunosorbent assay; FLSs: fibroblast-like synoviocytes; HA: hyaluronic acid; HUVEC: human umbilical vein endothelial cells; OA: osteoarthritis; PBS: phosphate-buffered saline; qPCR: quantitative real-time polymerase chain reaction; RA: rheumatoid arthritis; ROC: receiver operator characteristic; siRNA: small interfering RNA.}

\section{Competing interests}

The authors declare that they have no competing interests.

\section{Authors' contributions}

$P Q, B C, Y L, Z Z, R G$ and $H Z$ carried out the molecular genetic studies, JW and $X Y$ participated in the sequence alignment and drafted the manuscript. JW and PQ carried out the immunoassays and revised the manuscript. PQ and $\mathrm{BC}$ participated in the sequence alignment and drafted the manuscript JW and XY participated in the design of the study, performed the statistical analysis and helped to revise the manuscript. JW and JW conceived of the study, and participated in its design and coordination and helped to draft the manuscript. All authors read and approved the final manuscript. XY, PQ and $\mathrm{BC}$ made equal contributions to this work. All authors take responsibility for the integrity of the work.

\section{Acknowledgements}

Acknowledgements of Shanghai Changhai Hospital and Shanghai Guanghua Hospital providing clinical samples and Dr Marra for freely providing pcDNA3.2DEST-KIAA1199 (Institute of Molecular Cancer Research, University of Zurich, Zurich, Switzerland). This project was supported by the National Natural Science Foundation of China (Grant 81201375 and 81472055), Zhejiang College Student Innovative Research Team (Grant 2012 R413021 and 2013R413019), National college Students Innovative Entrepreneurial Training Program (Grant 201310343003) and The Natural Science Foundations of Zhejiang Province (Grant LR13H060001 and LY12H28003).

\section{Author details}

${ }^{1}$ Department of Biochemistry, School of Basic Medical Sciences, Wenzhou Medical University, Wenzhou, China. ${ }^{2}$ Department of Medicinal Chemistry, School of Pharmaceutical Sciences, Wenzhou Medical University, Wenzhou,
China. ${ }^{3}$ Department of Rheumatology, Jiamusi Central Hospital, Jiamusi, China.

Received: 18 December 2014 Accepted: 22 April 2015

Published online: 29 May 2015

\section{References}

1. Feldmann M, Brennan FM, Maini RN. Rheumatoid arthritis. Cell. 1996;85:307-10.

2. Sewell KL, Trentham DE. Pathogenesis of rheumatoid arthritis. Lancet. 1993;341:283-6.

3. Benito MJ, Murphy E, Murphy EP, van den Berg WB, FitzGerald O, Bresnihan B. Increased synovial tissue NF-kappa B1 expression at sites adjacent to the cartilage-pannus junction in rheumatoid arthritis. Arthritis Rheum. 2004;50:1781-7.

4. Miller MC, Manning HB, Jain A, Troeberg L, Dudhia J, Essex D, et al. Membrane type 1 matrix metalloproteinase is a crucial promoter of synovial invasion in human rheumatoid arthritis. Arthritis Rheum. 2009;60:686-97.

5. HUGE protein database. http://www.kazusa.or.jp/huge

6. Abe S, Usami S, Nakamura Y. Mutations in the gene encoding KIAA1199 protein, an inner-ear protein expressed in Deiters' cells and the fibrocytes, as the cause of nonsyndromic hearing loss. J Hum Genet. 2003;48:564-70.

7. He QY, Liu XH, Li Q, Studholme DJ, Li XW, Liang SP. G8: a novel domain associated with polycystic kidney disease and non-syndromic hearing loss. Bioinformatics. 2006:22:2189-91.

8. Guo J, Cheng H, Zhao S, Yu L. GG: a domain involved in phage LTF apparatus and implicated in human MEB and non-syndromic hearing loss diseases. FEBS Lett. 2006;580:581-4.

9. Matsuzaki S, Tanaka F, Mimori K, Tahara K, Inoue H, Mori M. Clinicopathologic significance of KIAA1199 overexpression in human gastric cancer. Ann Surg Oncol. 2009;16:2042-51.

10. Michishita E, Garces G, Barrett JC, Horikawa I. Upregulation of the KIAA1199 gene is associated with cellular mortality. Cancer Lett. 2006;239:71-7.

11. Kuscu C, Evensen N, Kim D, Hu YJ, Zucker S, Cao J. Transcriptional and epigenetic regulation of KIAA1199 gene expression in human breast cancer. PLoS One. 2012;7, e44661.

12. Evensen NA, Kuscu C, Nguyen HL, Zarrabi K, Dufour A, Kadam P, et al. Unraveling the role of KIAA1199, a novel endoplasmic reticulum protein, in cancer cell migration. J Natl Cancer Inst. 2013;105:1402-16.

13. Kita H, Hikichi Y, Hikami K, Tsuneyama K, Cui ZG, Osawa H, et al. Differential gene expression between flat adenoma and normal mucosa in the colon in a microarray analysis. J Gastroenterol. 2006:41:1053-63.

14. Sabates-Bellver J, Van der Flier LG, de Palo M, Cattaneo E, Maake C, Rehrauer $\mathrm{H}$, et al. Transcriptome profile of human colorectal adenomas. Mol Cancer Res. 2007;5:1263-75.

15. Tiwari A, Schneider M, Fiorino A, Haider R, Okoniewski MJ, Roschitzki B, et al. Early insights into the function of KIAA1199, a markedly overexpressed protein in human colorectal tumors. PLoS One. 2013 8:e69473.

16. LaPointe LC, Pedersen SK, Dunne R, Brown GS, Pimlott L, Gaur S, et al. Discovery and validation of molecular biomarkers for colorectal adenomas and cancer with application to blood testing. PLoS One. 2012 7:e29059.

17. Birkenkamp-Demtroder K, Maghnouj A, Mansilla F, Thorsen K, Andersen CL, Oster B, Hahn et al. Repression of KIAA1199 attenuates Wnt-signalling and decreases the proliferation of colon cancer cells. Br J Cancer. 2011 105:552-61.

18. leta K, Tanaka F, Haraguchi N, Kita Y, Sakashita H, Mimori K, et al. Biological and genetic characteristics of tumor-initiating cells in colon cancer. Ann Surg Oncol. 2008;15:638-48.

19. Wang JG, Xu WD, Zhai WT, Li Y, Hu JW, Hu B, et al. Disorders in angiogenesis and redox pathways are main factors contributing to the progression of rheumatoid arthritis: a comparative proteomics study. Arthritis Rheum. 2012;64:993-1004.

20. Salaffi F, Peroni M, Ferraccioli GF. Discriminating ability of composite indices for measuring disease activity in rheumatoid arthritis: a comparison of the Chronic Arthritis Systemic Index, Disease Activity Score and Thompson's articular index. Rheumatology (Oxford). 2000;39:90-6.

21. Arnett FC, Edworthy SM, Bloch DA, McShane DJ, Fries JF, Cooper NS, et al. The American rheumatism association 1987 revised criteria for the classification of rheumatoid arthritis. Arthritis Rheum. 1988;31:315-24.

22. Takigawa M, Enomoto M, Nishida Y, Pan HO, Kinoshita A, Suzuki F. Tumor angiogenesis and polyamines: alpha-difluoromethylornithine, an irreversible inhibitor of ornithine decarboxylase, inhibits B16 melanoma-induced 
angiogenesis in ovo and the proliferation of vascular endothelial cells in vitro. Cancer Res. 1990:50:4131-8.

23. Klareskog L, Catrina Al, Paget S. Rheumatoid arthritis. Lancet. 2009;373:659-72.

24. Yoshida H, Nagaoka A, Kusaka-Kikushima A, Tobiishi M, Kawabata K, Sayo T, et al. KIAA1199, a deafness gene of unknown function, is a new hyaluronan binding protein involved in hyaluronan depolymerization. Proc Natl Acad Sci USA. 2013 110:5612-17.

25. Yoshida H, Nagaoka A, Nakamura S, Sugiyama Y, Okada Y, Inoue S. Murine homologue of the human KIAA1199 is implicated in hyaluronan binding and depolymerization. FEBS Open Bio. 2013 3:352-56

26. Yoshida H, Nagaoka A, Nakamura S, Tobiishi M, Sugiyama Y, Inoue S. $\mathrm{N}$-Terminal signal sequence is required for cellular trafficking and hyaluronan-depolymerization of KIAA1199. FEBS Lett. 2014 588:111-16.

27. Miao CG, Yang YY, He X, Li XF, Huang C, Huang Y, et al. Wht signaling pathway in rheumatoid arthritis, with special emphasis on the different roles in synovial inflammation and bone remodeling. Cell Signal. 2013 25:2069-2078.

28. Jami MS, Hou J, Liu M, Varney ML, Hassan H, Dong J, et al. Functional proteomic analysis reveals the involvement of KIAA1199 in breast cancer growth, motility and invasiveness. BMC Cancer. 2014 14:194.

29. Nakayama M, Kikuno R, Ohara O. Protein-protein interactions between large proteins: two-hybrid screening using a functionally classified library composed of long cDNAs. Genome Res. 2002;12:1773-84.

30. Rujescu D, Meisenzahl EM, Krejcova S, Giegling I, Zetzsche T, Reiser M, et al. Plexin B3 is genetically associated with verbal performance and white matter volume in human brain. Mol Psychiatry. 2007;12:190-4. 115.

31. Balakrishnan A, Penachioni JY, Lamba S, Bleeker FE, Zanon C, Rodolfo M, et al. Molecular profiling of the "plexinome" in melanoma and pancreatic cancer. Hum Mutat. 2009;30:1167-74.

32. Roth L, Koncina E, Satkauskas S, Cremel G, Aunis D, Bagnard D. The many faces of semaphorins: from development to pathology. Cell Mol Life Sci. 2009;66:649-66.

33. Bradham DM, Igarashi A, Potter RL, Grotendorst GR. Connective tissue growth factor: a cysteine-rich mitogen secreted by human vascular endothelial cells is related to the SRC-induced immediate early gene product CEF-10. J Cell Biol. 1991;114:1285-94.

34. Lin L, Lesnick TG, Maraganore DM, Isacson O. Axon guidance and synaptic maintenance: preclinical markers for neurodegenerative disease and therapeutics. Trends Neurosci. 2009;32:142-9.

35. Sadanandam A, Varney ML, Singh S, Ashour AE, Moniaux N, Deb S, et al. High gene expression of semaphorin 5A in pancreatic cancer is associated with tumor growth, invasion and metastasis. Int J Cancer. 2010;127:1373-83.

36. Fiore R, Rahim B, Christoffels VM, Moorman AF, Puschel AW. Inactivation of the Sema5a gene results in embryonic lethality and defective remodeling of the cranial vascular system. Mol Cell Biol. 2005;25:2310-9.

37. Sadanandam A, Rosenbaugh EG, Singh S, Varney M, Singh RK. Semaphorin $5 \mathrm{~A}$ promotes angiogenesis by increasing endothelial cell proliferation, migration, and decreasing apoptosis. Microvasc Res. 2010;79:1-9.

\section{Submit your next manuscript to BioMed Central and take full advantage of:}

- Convenient online submission

- Thorough peer review

- No space constraints or color figure charges

- Immediate publication on acceptance

- Inclusion in PubMed, CAS, Scopus and Google Scholar

- Research which is freely available for redistribution

Submit your manuscript at www.biomedcentral.com/submit 